$+{ }^{+}+{ }^{+}+$La revue pour l'histoire du CNRS

POUR L LHISTOIRE DU CNRS $\quad 24 \mid 2009$

Soixante-dixième anniversaire du CNRS

\title{
Le CNRS, un Européen convaincu
}

Pierre Papon

\section{(2) OpenEdition}

Journals

Édition électronique

URL : https://journals.openedition.org/histoire-cnrs/9144

DOI : 10.4000/histoire-cnrs.9144

ISSN : 1955-2408

Éditeur

CNRS Éditions

Édition imprimée

Date de publication : 5 octobre 2009

ISSN : 1298-9800

Référence électronique

Pierre Papon, « Le CNRS, un Européen convaincu », La revue pour l'histoire du CNRS [En ligne], 24 | 2009, mis en ligne le 05 octobre 2009, consulté le 20 mai 2021. URL : http://journals.openedition.org/ histoire-cnrs/9144; DOI : https://doi.org/10.4000/histoire-cnrs.9144

Ce document a été généré automatiquement le 20 mai 2021.

Comité pour l'histoire du CNRS 


\title{
Le CNRS, un Européen convaincu
}

\author{
Pierre Papon
}

1 Il y a cinquante ans, la coopération scientifique est d'abord apparue à l'Europe comme un moyen privilégié pour fédérer ses forces dans des domaines de la science comme la physique des particules et l'astronomie où de grandes infrastructures étaient nécessaires pour la recherche. Cette coopération permet aussi, aujourd'hui, de mettre en oeuvre de grands programmes de recherche, de mener à bien des projets de longue durée en mettant en commun des moyens et des méthodes tout en favorisant la mobilité des chercheurs.

2 Le CNRS a été associé, directement ou indirectement, à la création de l'Europe de la recherche dès ses débuts. Il faut ainsi rappeler le rôle clé qu'a joué le physicien Pierre Auger dans la création du Cern, en 1953, étape marquante de la coopération scientifique européenne (celui-ci dirigea plusieurs laboratoires du CNRS après la guerre). Le Cern est devenu le point focal mondial de la physique des hautes énergies et les laboratoires de l'IN2P3 participent activement à la préparation des expériences qui seront réalisées, après 2009, avec le nouvel accélérateur, le Large Hadron Collider (LHC), qui est pour les physiciens le sésame qui doit leur ouvrir la voie vers la découverte de nouvelles particules; l'IN2P3 a aussi contribué à la conception des aimants supraconducteurs de cette nouvelle machine. En astronomie, de même, les équipes CNRS sont associées aux grands projets d'exploration de l'Univers tant avec les télescopes de l'Eso (European Southern Observatory) qu'à travers des missions spatiales de l'Esa (certaines d'entre elles participent ainsi aux missions spatiales de l'Esa Planck mesure du rayonnement fossile de l'Univers - et Herschel).

Plus directement, le CNRS a joué un rôle de pionnier aux côtés de ses partenaires européens dans la construction de grands instruments européens, dont l'Institut LaueLangevin (ILL) et l'ESRF (European Synchrotron Radiation Facility) à Grenoble sont les figures de proue. Il participe ainsi directement à la gestion d'une demi-douzaine d'infrastructures européennes avec un nombre variable de partenaires (avec le CEA pour l'ILL et l'ESRF) : l'Allemagne et l'Espagne pour l'Iram en astronomie millimétrique, l'Italie avec l'installation du laser Virgo pour détecter les ondes gravitationnelles, etc. 
4 La coopération européenne, c'est aussi une aventure quotidienne pour les laboratoires du CNRS impliqués dans les très nombreux projets menés avec des laboratoires d'autres pays européens sous l'égide des Programmes cadres de recherche développement (PCRD) de l'Union européenne et cela depuis plus de vingt ans.

5 Ainsi de 2000 à 2006, le CNRS, avec ses laboratoires propres et ses laboratoires mixtes, a été le premier organisme européen bénéficiaire des financements du sixième PCRD. Dans les sciences humaines et sociales, les équipes du CNRS ont joué un rôle moteur dans plusieurs programmes européens qui permettent de constituer des bases de données à l'échelle de l'Europe pour mieux comprendre les évolutions de nos sociétés (tel l'European Social Survey lancé à l'initiative de la Fondation européenne de la science destiné à repérer les changements des valeurs sociales). Plus récemment, des laboratoires du CNRS ont joué un rôle de pionnier en participant à la création de réseaux d'excellence européens qui ont pour objectif d'intégrer sur une longue durée des équipes européennes travaillant sur des thématiques communes. Plusieurs réseaux en science des matériaux coordonnés par des laboratoires du CNRS ont créé, en 2008, des structures européennes pérennes pour atteindre une masse critique de recherche dans leur domaine et organiser des enseignements de formation doctorale à l'échelle de l'Europe (par exemple l'Institut européen des matériaux multifonctionnels dans le domaine des matériaux céramiques et hybrides piloté par un laboratoire de Bordeaux).

6 Au fil des décennies, le CNRS a apporté sa pierre à la construction de l'Espace européen de la recherche à travers les grandes organisations et programmes européens : il a joué un rôle clé dans la création, en 1974, de la Fondation européenne de la science (ESF) dont Hubert Curien fut l'un des présidents. Gérard Mégie, pour sa part, a contribué à promouvoir l'idée de créer le Conseil européen de la recherche (ERC), ses laboratoires ont animé avec leur savoir-faire des grands projets européens (comme Epica pour les forages de glace dans l'Antarctique afin de restituer les climats du passé, prix Descartes 2008 pour la recherche européenne). Le CNRS a donc été présent sur tous les fronts de la recherche européenne.

7 La longue histoire de la coopération scientifique européenne montre bien que l'Europe est parvenue à maintenir son rang dans la recherche internationale en unissant le savoir-faire de ses laboratoires et en mettant en commun des moyens financiers et techniques. Tout en développant son propre potentiel et ses implantations dans les régions françaises, le CNRS a participé activement à cette politique européenne, car il avait conscience que la recherche était une entreprise internationale dont la dimension européenne devait prendre une importance croissante. La compétitivité scientifique future de l'Europe dépend de sa capacité à unir encore davantage ses forces pour constituer un pôle d'attraction mondial, c'est ce que montre l'expérience du Cern et de plusieurs programmes européens, en particulier pour les jeunes scientifiques d'autres continents. À cet égard, l'ERC est une pièce fondamentale pour l'avenir de la recherche européenne dans la mesure où il renforcera son excellence, un dixième des projets financés par le Conseil en 2008 ayant profité à des jeunes chercheurs du CNRS.

8 On peut envisager un scénario à long terme où l'ERC deviendrait une véritable agence de la recherche fondamentale européenne travaillant en association étroite avec les agences nationales comme le CNRS. On doit aussi souhaiter que le CNRS continue à jouer son rôle de catalyseur pour que se constitue un large réseau d'infrastructures de recherche à dimension européenne (machines, calculateurs, bases de données en sciences sociales et en génétique, etc.). L'Espace européen de la recherche permet au 
CNRS d'amplifier ses propres efforts de recherche grâce aux synergies qu'il permet d'établir avec ses partenaires européens tout en participant à un effort international pour relever quelques grands défis auxquels est confrontée la planète: explorer l'Univers et la matière, percer les secrets du vivant, mieux comprendre l'évolution du climat, découvrir de nouveaux modes de production et d'utilisation de l'énergie, etc. N'oublions pas non plus que la coopération scientifique européenne dans les années cinquante se voulait une contribution à l'effort de reconstruction de l'Europe; en multipliant les échanges entre les chercheurs et en créant peu à peu un maillage entre les laboratoires européens dans toutes les disciplines, la recherche a sans aucun doute activement contribué à la construction européenne et le CNRS a été l'un des acteurs de cette entreprise qui est loin d'être achevée.

9 L'Espace européen de la recherche peut, à l'avenir, aider l'Europe à mieux comprendre ses origines historiques ainsi que sa diversité culturelle tout en lui apportant une vision prospective de son avenir : à quels défis doit-elle faire face et comment? Fort de son expérience, le CNRS, avec ses partenaires français et européens, peut contribuer à faire de l'Espace européen de la recherche, une réalité.

1. Datant du Crétacé (environ 100 millions d'années), guêpe fossile préservée dans de l'ambre opaque et provenant d'un gisement en Charente (France). Sa découverte, et son image ont été rendues possible grâce à la technique de l'imagerie en contraste de phase utilisant le rayonnement synchrotron et développée à l'ESRF (European Synchrotron Radiation Facility), à Grenoble. L'animal mesure $4 \mathrm{~mm}$ environ et cette image révèle des détails d'ordre micrométrique. (c) CNRS Photothèque/ESRF/ Malvina Lak, Didier Neraudeau, Paul Tafforeau

11 2. Dôme Concordia en Antarctique: montage de la tente de forage Epica (European Project for Ice Coring in Antarctica) dite « la cathédrale ». () CNRS Photothèque/Laurent Augustin

12 3. L'expérience Virgo. Ici, le tunnel nord, le 20 septembre 2000, à Cascina, près de Pise (Italie). À l'arrière plan, on distingue le premier pont et le bâtiment d'assemblage du tube. (C) CNRS Photothèque/EGO/Daniel Enard

\section{RÉSUMÉS}

La recherche scientifique a été et reste une grande aventure collective en Europe, souvent en dépit des crises que notre continent traversait, mais ce n'est qu'après la seconde guerre mondiale que la coopération scientifique européenne a pris une forme institutionnelle alors que le CNRS lui-même s'organisait et se développait. Le CNRS, un acteur majeur de l'Espace européen de la recherche? «Oui ! », répond sans hésiter Pierre Papon, ancien directeur général du CNRS. 
AUTEUR

PIERRE PAPON

Pierre Papon, ancien directeur général du CNRS, est professeur émérite à l'École supérieure de physique et chimie industrielles de la Ville de Paris (ESPCI). 